\title{
Explaining the Increasing Heritability of Cognitive Ability Across Development: A Meta-Analysis of Longitudinal Twin and Adoption Studies
}

\author{
Daniel A. Briley and Elliot M. Tucker-Drob \\ Department of Psychology and Population Research Center, University of Texas at Austin
}

\begin{abstract}
Genes account for increasing proportions of variation in cognitive ability across development, but the mechanisms underlying these increases remain unclear. We conducted a meta-analysis of longitudinal behavioral genetic studies spanning infancy to adolescence. We identified relevant data from 16 articles with 11 unique samples containing a total of 11,500 twin and sibling pairs who were all reared together and measured at least twice between the ages of 6 months and 18 years. Longitudinal behavioral genetic models were used to estimate the extent to which early genetic influences on cognition were amplified over time and the extent to which innovative genetic influences arose with time. Results indicated that in early childhood, innovative genetic influences predominate but that innovation quickly diminishes, and amplified influences account for increasing heritability following age 8 years.
\end{abstract}

\section{Keywords}

behavior genetics; cognition; intelligence; development; amplification; childhood development; cognitive ability

Children are born with all of their genes, and their experiences necessarily accrue as they develop. ${ }^{1}$ It is therefore reasonable to expect that genetic variation will account for increasingly less variation in psychological outcomes as children develop, and variation in environmental experiences will account for increasingly more of the variation in psychological outcomes (e.g., see Fryer \& Levitt, 2006; Spelke, 2005; cf. McGue, Bouchard, Iacono, \& Lykken, 1993). One might expect this rationale to apply particularly well to a highly complex psychological outcome, such as cognition, which is thought to develop as a result of experience-dependent neural connections (Garlick, 2002) and is known to depend on environmental quality and educational experience (Ceci, 1991; Nelson et al., 2007). Somewhat counterintuitively, a number of studies have indicated precisely the

(C) The Author(s) 2013

Corresponding Author: Daniel A. Briley, Department of Psychology, University of Texas at Austin, 108 E. Dean Keeton Stop A8000, Austin, TX 78712-1043 daniel.briley@utexas.edu.

Author Contributions: D. A. Briley primarily analyzed the data, interpreted results, and prepared the manuscript under the supervision of E. M. Tucker-Drob, who additionally shaped the study concept and made substantial contributions to the final manuscript. Both authors approved the final version of the manuscript for submission.

Declaration of Conflicting Interests: The authors declared that they had no conflicts of interest with respect to their authorship or the publication of this article.

Supplemental Material: Additional supporting information may be found at http://pss.sagepub.com/content/by/supplemental-data

${ }^{1}$ We refer here to objective experiences, defined as "environmental events as they might be observed by a researcher, as opposed to how they affect family members" (Turkheimer \& Waldron, 2000, p. 79). 
opposite developmental pattern with respect to the genetic and environmental influences on cognition. McCartney, Harris, and Bernieri (1990) provided some of the earliest, persuasive evidence of this trend. In their analysis of 103 twin studies, the amount of variation attributable to genetic differences correlated positively with the age of the twins $(r=.36)$, and the amount of variation attributable to the shared rearing environment correlated negatively $(r=-.37)$. More recently, Bergen, Gardner, and Kendler (2007) conducted a meta-analysis of six studies and reported an increase in heritability from approximately $55 \%$ at age 13 years to $70 \%$ at age 25 years. Haworth et al. (2010) synthesized individual-level data from six studies containing information from 11,000 twin pairs and found that heritability increased from $41 \%$ at age 9 years to $66 \%$ at age 17 years.

The mechanisms that give rise to these developmental increases in heritability remain poorly understood. Two possibilities are what can be termed innovation and amplification (Plomin, 1986; Plomin \& DeFries, 1985). Innovation refers to the possibility that increasing heritability results from novel genetic influences that were not present at previous time points. Innovation might arise because novel biological changes (e.g., hormonal changes associated with puberty) or environmental changes (e.g., the transition from the home to grade school) lead to the activation of genes. Compounded with previously active genetic variation, this newly active genetic variation can lead to an increase in heritability.

Amplification refers to the possibility that early genetic influences on cognition become increasingly important with age. For example, transactional processes in which children select and evoke environments on the basis of small, genetically influenced differences in ability may, in turn, magnify those differences (Dickens \& Flynn, 2001). Of course, decay processes may also operate, such that early genetic influences may not persist at full strength over time.

It is necessary to combine behavioral genetic and developmental methodologies to distinguish between innovation and amplification processes as the basis for increasing heritability. The classic twin model decomposes variation in cognitive test scores into proportions accounted for by genes $(A)$; the shared environment $(C)$, which operates at the family level and serves to make children living in the same household more similar; and the nonshared environment $(E)$, which operates at the individual level and serves to differentiate children living in the same household. This analysis hinges on whether more genetically similar siblings are also more similar in their level of cognitive ability (Neale \& Cardon, 1992). Longitudinal designs allow variation in an outcome to be partitioned into that which is shared with an earlier time point and that which is unique to a later time point.

Longitudinal behavioral genetic models allow the variance in intelligence at each time point as well as the stable variance between time points to be decomposed into that due to $A, C$, and $E$. The increase in heritability of cognition can be understood in terms of the net effects of increases attributable to amplification (or decreases due to decay) of previously active genetic influences and those attributable to innovative, not previously active, genetic influences.

\section{Goal of the Present Study}

Our goal in the present study was to examine how genetic and environmental influences on cognition change over time by conducting a meta-analysis of data from longitudinal twin and adoption studies of cognitive ability in children aged 6 months to 18 years. First, we sought to replicate the trend of increasing heritability using only longitudinal data, which avoids validity threats associated with cross-sectional approaches (e.g., cohort effects; Baltes, 1968; Flynn, 1987). For example, if a study tested children at age 5 years and again at age 10 years, we examined the change accounted for by genetic and environmental factors over the 5-year interval, controlling for the age of assessment. Second, to explain these 
developmental changes, we examined age trends in amplification and innovation, controlling for the time interval between measurements.

\section{Method}

We searched abstracts in the American Psychological Association's PsycINFO database (http://www.apa.org/pubs/databases/psycinfo/index.aspx) using combinations of terms from three categories: genetics (twin, genetic, adoption, adopted, adoptee), longitudinal (longitudinal, aging, stability), and cognition (intelligence, cognition, cognitive, ability). We included studies with the following characteristics: The samples of siblings had varying degrees of genetic relatedness, there was enough information to derive complete across-time and within-time sibling correlations, the same ability was measured on two or more occasions using objective cognition/intelligence tests, and participants were age 18 years or younger at baseline and at least one follow-up occasion. Full information concerning our search process, the articles included, study composition, data extraction, and variables analyzed can be found in the Supplemental Material available online. We identified effect sizes from 16 articles and 11 unique samples containing longitudinal information from 4,047 monozygotic twin, 7,169 dizygotic twin, 141 adoptive sibling, and 143 nonadoptive sibling pairs. All studies used a reared-together design. For each longitudinally measured cognitive outcome from each study, we compiled within- and across-time sibling correlations, separately by sibling type.

We used Mplus software (Muthén \& Muthén, 2010) to specify longitudinal Cholesky decompositions to the multigroup correlation matrices. Figure 1 presents a reduced version of the Cholesky decomposition for one member of a sibling pair. Cognitive ability is partitioned into $A, C$, and $E$ sources of variance. The latent variables labeled $A_{1}, C_{2}$, and $E_{1}$ represent these influences on cognition at Time 1 , and squaring the parameters $a_{1}, c_{1}$, and $e_{1}$ gives the proportion of variance in cognition at Time 1 accounted for by the $A_{1}, C_{1}$, and $E_{1}$ components, respectively. The parameters labeled $a_{b}, c_{b}$, and $e_{b}$ represent the carryover of genetic and environmental effects from Time 1 to Time 2. Amplification of genetic effects was calculated by taking the difference between the squared $a_{1}$ and $a_{b}$ parameters, with positive values indicating amplification and negative values indicating decay. Finally, the latent variables $A_{u}, C_{u}$, and $E_{u}$ represent the unique variance at the second time point, and the squared $a_{u}, c_{u}$, and $e_{u}$ parameters represent the proportion of variance attributable to innovation. Proportions of variance in cognition at Time 2 attributable to $A, C$, and $E$ were computed as the sum of the squares of $a_{b}$ and $a_{u}, c_{b}$ and $c_{u}$, and $e_{b}$ and $e_{u}$, respectively. Across each pair of longitudinal observations, we recorded changes in proportions of variance attributable to $A, C$, and $E$ and the amounts of amplification and innovation of $A, C$, and $E .^{2}$ In total, our longitudinal analyses made use of 125 unique pairs of repeated measures, yielding $125 \times 9$ effect sizes. Each effect size was associated with information regarding participants' age when assessed, the length of time between measurement occasions, and the standard error of the estimate.

To estimate the influence of development on the variance components, we applied a series of meta-analytic, random-effects models. Not only do random-effects models index the imprecision of the meta-analytic estimates (i.e., the standard error of the meta-analytic parameter), but they also index the variability of the true effect size across studies. This is

\footnotetext{
${ }^{2}$ To be more explicit about how each effect size was derived, we report the mathematical calculations. For genetic factors, change in the proportion of variance was calculated as $\left(a_{b}^{2}+a_{u}^{2}\right)-a_{1}^{2}$, amplification was calculated as $a_{b}^{2}-a_{1}^{2}$, and innovation was
calculated as $a_{u}$. For shared environmental factors, change in the proportion of variance was calculated as $\left(c_{b}^{2}+c_{u}^{2}\right)-c_{1}{ }^{2}$, amplification was calculated as $c b^{2}-c 1^{2}$, and innovation was calculated as $c_{u}^{2}$. For nonshared environmental factors, change in the proportion of variance was calculated as $\left(e_{b}^{2}+e_{u}^{2}\right)-e_{1}^{2}$, amplification was calculated as $e_{b}^{2}-e_{1}^{2}$, and innovation was calculated as $e_{u}^{2}$.
} 
advantageous because it allows the results of the model to generalize beyond the studies that were included in the analysis to a theoretical population of heterogeneous studies (Hedges \& Vevea, 1998). We adopted the general approach described by Cheung (2008) to estimate meta-analytic, random-effects models in Mplus. A weighting procedure was applied to provide unbiased standard errors. This entailed weighting all variables in our analysis by the inverse of the sampling variance of the dependent variable and the inverse of the number of effect sizes included per study. Additionally, the cluster option of Mplus was applied to correct for nonindependence of data points taken from the same study. Together, these corrections ensured that our standard errors accurately reflected the precision of the original estimates and were not biased as a result of including multiple effect sizes per study.

To ensure that results were not driven by studies with large sample sizes, we constructed Alternative Model 1 to examine whether results persisted when excluding results based on the very large Twins Early Development Study (TEDS). Further, in Alternative Model 2, we investigated whether results persisted when not weighting by precision of the estimates. We chose to exclude the TEDS data for comparison because it is by far the largest twin study of cognitive development in this age range (Davis, Haworth, \& Plomin, 2009). Therefore, this data set would have had substantially more leverage on the results than other studies, and we were interested in whether the identified trends held when effect sizes from this study were excluded. Additionally, this study used a unique modeling approach that may have influenced the extracted data. Rather than evaluating a model that included multiple indicators of cognition at a given time point, Davis et al. (2009) constructed a model that included cognition at multiple time points to form each latent variable. This may have influenced the comparability of this study with others included in the meta-analysis.

Using these procedures, we ran a series of meta-analytic, random-effects regression models that predicted the nine outcomes of interest from the age of the participants at the initial time point, the time interval between measurements, and the estimate of the magnitude of heritability or environmentality (i.e., the proportion of variance due to environmental effects) at the initial time point. Age and time interval between measurements were centered at their across-sample averages ( 4.22 and 4.31 years, respectively). The initial estimates of genetic and environmental factors were centered at values expected based on the acrosssample average age $\left(a^{2}=.36, c^{2}=.43, e^{2}=.22\right)$. We had two main empirical questions. First, does the trend of increasing heritability hold when longitudinal data are used to examine differences in time lag rather than age? Second, across the developmental period of infancy to adolescence, do innovative or amplified genetic effects explain increases in heritability? In our analyses of change in the magnitudes of $a^{2}, c^{2}$, and $e^{2}$, our main emphasis was on interpreting the time-interval parameter to verify that heritability increases over time, even with participant age controlled. In our analyses of amplification and innovation, our main emphasis was on interpreting the age parameter with time interval between measurements controlled.

\section{Results \\ Descriptive statistics}

Table 1 presents characteristics of samples in the studies included in the meta-analysis. The studies covered the entire span from 6 months to 18 years of age, with substantial variability in longitudinal time intervals between measurements. There was a fairly even split between male and female individuals, but the studies predominantly sampled White participants. 


\section{Changes in genetic and environmental factors}

Previous reports of developmental increases in heritability were largely confirmed by our longitudinal meta-analysis of within-study change presented in Table 2. Longer time intervals between measurements were associated with larger increases in heritability $(b=$ $0.028, p<.01)$ and larger decreases in shared environmentality $(b=-0.038, p<.001)$. Larger increases in heritability were also associated with older ages $(b=0.023, p<.01)$. The results for the developmental trends in the nonshared environment were slightly more complex. Longer longitudinal time intervals between measurements were associated with larger increases in the variance attributable to the nonshared environment $(b=0.019, p<$. 001 ), and older age was associated with smaller increases in the variance attributable to the nonshared environment $(b=-0.011, p<.05)$. The trends for the effects of time interval between measurements are plotted in Figure 2 over a range of 15 years, the longest observed time interval in our meta-analysis. Because participant age was controlled, the increases in heritability and decreases in shared environmentality evident in Figure 2 are entirely reflective of longitudinal changes, as opposed to cross-sectional age differences.

The results were similar in the alternative models, which indicate more pronounced increases in heritability and more pronounced decreases in shared environmentality over longer time intervals. Trends for the effects of time interval between measurements indicated by each of these alternative models are plotted in Figures S1 and S2 in the Supplemental Material.

\section{Amplification and innovation}

Table 3 presents the results of the random-effects regression models for the amplification of $A, C$, and $E$. Focusing on the primary model, we found that the intercept of each variance component was significantly negative. This indicates that earlier genetic or environmental effects have a tendency to decay, at least at this very early stage of development. Only one parameter was statistically significant by traditional standards, and it indicated that earlier shared environmental influences become less important with greater time lag. However, there were some potentially important trends that did not meet traditional levels of statistical significance. The age coefficients were marginally significant for genes $(b=0.089, p=.06)$ and the shared environment $(b=0.018, p=.07)$. Further, the age coefficient for genes was highly significant in both alternative models, and the age coefficient for the shared environment was significant when the TEDS data were removed.

As seen in Table 4, innovative influences displayed many strong associations with age and time lag. Focusing on the primary model, we found that the intercept of each variance component was significantly positive, and the coefficient for age was significantly negative. This indicates that novel genetic and environmental influences are more common early in life but diminish in importance with age. Longer time intervals between measurements were associated with a larger degree of innovative genetic and nonshared environmental influences but a smaller amount of innovative shared environmental influences. The results were generally attenuated in the alternative models, but there was only a small degree of absolute change in the coefficients.

To answer the question of what processes account for the changes in heritability and environmentality across the age range of 6 months to 18 years, we plotted the amplification and innovation trends against baseline age for each variance component, assuming a 1-year interval between measurement occasions (see Fig. 3). In the very early years of life, innovative genetic influences appear to account for the increase in heritability. By approximately age 8 , genetic amplification effects become predominant and innovative genetic effects reach zero. However, the primary model did not estimate the genetic 
amplification parameter very precisely, as is evident by the wide shading, rendering interpretation somewhat difficult. The alternative models imply a more gradual slope but with much greater precision. For the shared environment, innovative influences slowly decrease and decay effects slowly fade with age. Finally, the nonshared environment largely displayed decay effects that were replaced by innovative influences. Results from the alternative models are plotted in Figures S3 and S4 in the Supplemental Material.

\section{Discussion}

Despite the intuitive appeal of the prediction that accruing socializing forces result in decreased genetic influences on cognition with age, or that genetic influences are immutable and entirely apparent at birth, the field of developmental behavior genetics has uncovered evidence that the genetic influences on intelligence increase with age (Plomin \& Spinath, 2004). This integrative meta-analysis has attempted to provide an in-depth investigation of the mechanisms underlying this phenomenon. We focused on two types of processes that might underlie these changes, namely amplification, whereby early genetic influences on cognition carry over across time, and innovation, whereby novel genetic influences on cognition emerge with time. We found that each process varies systematically over the age range from infancy to adolescence. Put succinctly, genetic innovation predominates in infancy and early childhood, whereas genetic amplification predominates in middle childhood and adolescence. We discuss the implications of each of these trends below.

\section{Innovation}

In early childhood, increasing genetic influences on cognitive ability can be attributed to innovative genetic influences. In other words, genes not previously affecting cognition at one point in time begin to affect cognition at later points in time. What may be the causes of this early innovation? One possible mechanism of innovation is that biological maturation across infancy and early childhood may activate genes. Although puberty is an often cited example of a developmental transition that may be accompanied by the preprogrammed activation of genetic influences (Eaves, Long, \& Heath, 1986), we found that innovative genetic influences predominate well before the earliest beginning ages of adrenarche (McClintock \& Herdt, 1996). A second possible mechanism for innovation may be children's continual introduction into new environments that activate genes for cognition. This can occur through experience-dependent activation of genes at the level of the genome, but it can also occur through a process by which early genetically influenced traits irrelevant to cognition in home or day-care contexts become progressively more important for cognitive development in kindergarten and grade school contexts (Tucker-Drob \& Harden, 2012b).

Innovative shared environmental effects are also present but slowly fade in importance. One possible explanation for this trend is the introduction into the educational system. Innovative shared environmental effects might occur as some families send their children to day care and preschool, whereas other families provide early care at home. However, as all children ultimately enter the educational system, standardized educational practices can serve to equalize differences between families (Downey, von Hippel, \& Broh, 2004; Tucker-Drob, 2012).

\section{Amplification}

In early childhood, decay, not amplification, of earlier genetic and environmental influences appears to be the rule rather than the exception. These trends change with age, such that by middle childhood, genetic influences stop decaying and become amplified. Transactional models provide an appealing framework for understanding amplification. Under 
transactional models, genetic influences become amplified through a process in which early levels of ability become reinforced through the selection and evocation of experiences consistent with those ability levels (Dickens \& Flynn, 2001; Plomin, DeFries, \& Loehlin, 1977). Scarr and McCartney (1983) suggested that one's genotype drives development, because "it is the discriminator of what environments are actually experienced" (p. 425). For example, small genetically influenced differences in cognitive ability can lead to differences in aspects of the environment provided by parents, teachers, and peers that in turn influence subsequent cognitive development (Tucker-Drob \& Harden, 2012a, 2012b).

\section{Strengths and limitations}

A major strength of the current study is that we applied a standard behavioral genetic model to data derived from different empirical reports to yield a common set of parameters that could be aggregated. This was particularly advantageous in allowing us to combine data from studies of different age periods to make inferences about the entire range of development from infancy to adolescence. In total, our meta-analysis was derived from objective assessments of more than 23,000 individuals. However, this sample was obtained from a relatively small number of independent longitudinal samples. Our estimates were therefore likely to be vulnerable to idiosyncratic aspects of individual studies. For example, when the TEDS data were removed, the parameter estimate for the amplification of genetic effects was far more precise. This may be due to the unique modeling approach taken by the original authors or to potential differences in developmental processes across populations (e.g., Hanscombe et al., 2012). However, parameter values (as separate from significance levels) across primary and alternative models were remarkably consistent.

A second limitation is that we were unable to determine the extent to which the nonshared environment represented true environmental influences rather than error of measurement. Although the nonshared environment can be corrected of measurement error with information regarding test reliability, this was not well reported in the studies included in our meta-analysis. Therefore, we must assume that the level of measurement error is not systematically confounded with age to draw conclusions about age trends in the nonshared environment.

A third limitation is that we were able only to compute standardized estimates of genetic and environmental contributions. Longitudinal covariance matrices (as opposed to correlation matrices) of the same measures taken over time are necessary to estimate unstandardized genetic and environmental variance components and evaluate changes in total phenotypic variance with age. A minority of studies (25\%) reported enough information to produce usable covariance rather than correlational matrices. This amounted to an even smaller portion of extracted sets of effect sizes (13.6\%), rendering exploration of changes in amounts (rather than proportions) of variance attributable to genetic and environmental amplification and innovation severely limited.

A fourth limitation is that the cognitive tests were often upgraded to be developmentally appropriate across waves. This was the case for half of the studies and the majority of extracted sets of effect sizes (64.8\%; see Table S1 in the Supplemental Material for details). If changing tests resulted in changing the abilities that were measured, our innovation estimates could be inflated. We avoided this threat by including only studies that measured the same ability over time (even if assessed with different measures). Empirically, we evaluated this influence in our primary models by including a dummy-coded variable indicating whether or not the cognitive test had changed between time points. This parameter was not significant for any model of amplification, but it was significant for models of genetic and nonshared environmental innovation. However, for both of these 
models, the age parameter, which was the focal parameter in this analysis, remained statistically significant and was altered only by a value of .001 .

Finally, we focused on linear models of developmental change. However, linear models are simplifications of more complex trends that are likely to occur across development. As part of the current project, we had also fit quadratic age functions to the genetic amplification and innovation effect sizes, but the quadratic terms were not significant. More studies are likely required for precise nonlinear age trends in innovation and amplification to be accurately estimated with meta-analysis.

\section{Conclusion}

By applying longitudinal behavioral genetic models to meta-analytic data on twins and siblings, we sought to identify the mechanisms underlying the well-established finding that genetic influences on cognitive abilities increase over the course of development (Bergen et al., 2007; Haworth et al., 2010). Our results indicate that longitudinal changes in heritability can be understood in terms of both innovative variance explained by genes not previously active and carryover (amplification and decay) of previously active genetic influences, with the relative contributions of each of these mechanisms differing across development. During the early years of life, it appears that genes are "activated," whereas previous genetic influences decay. These relative contributions change gradually, such that by approximately 8 years of age, genetic innovation desists and existing genetic influences begin to amplify.

\section{Supplementary Material}

Refer to Web version on PubMed Central for supplementary material.

\section{Acknowledgments}

Funding: This research was supported by National Institutes of Health Grant R21HD069772. The Population Research Center is supported by National Institutes of Health Grant R24HD042849. Daniel A. Briley was supported as a trainee by National Institutes of Health Grant T32HD007081.

\section{References}

Baltes PB. Longitudinal and cross-sectional sequences in the study of age and generation effects. Human Development. 1968; 11:145-171. [PubMed: 5663535]

Bergen SE, Gardner CO, Kendler KS. Age-related changes in heritability of behavioral phenotypes over adolescence and young adulthood: A meta-analysis. Twin Research and Human Genetics. 2007; 10:423-433. [PubMed: 17564500]

Ceci SJ. How much does schooling influence general intelligence and its cognitive components? A reassessment of the evidence. Developmental Psychology. 1991; 27:703-722.

Cheung MWL. A model for integrating fixed-, random-, and mixed-effects meta-analyses into structural equation modeling. Psychological Methods. 2008; 13:182-202. [PubMed: 18778151]

Davis OSP, Haworth CMA, Plomin R. Dramatic increase in heritability of cognitive development from early to middle childhood: An 8-year longitudinal study of 8,700 pairs of twins. Psychological Science. 2009; 20:1301-1308. [PubMed: 19732386]

Dickens WT, Flynn JR. Heritability estimates versus large environmental effects: The IQ paradox resolved. Psychological Review. 2001; 108:346-369. [PubMed: 11381833]

Downey DB, von Hippel PT, Broh BA. Are schools the great equalizer? Cognitive inequality during the summer months and the school year. American Sociological Review. 2004; 69:613-635.

Eaves LJ, Long J, Heath AC. A theory of developmental change in quantitative phenotypes applied to cognitive development. Behavior Genetics. 1986; 16:143-162. [PubMed: 3707482] 
Flynn JR. Massive IQ gains in 14 nations: What IQ tests really measure. Psychological Bulletin. 1987; 101:171-191.

Fryer, RG.; Levitt, S. Testing for racial differences in the mental ability of young children (NBER Working Paper No 12066). Cambridge, MA: National Bureau of Economic Research; 2006.

Garlick D. Understanding the nature of the general factor of intelligence: The role of individual differences in neural plasticity as an explanatory mechanism. Psychological Review. 2002; 109:116-136. [PubMed: 11863034]

Hanscombe KB, Trzaskowski M, Haworth CMA, Davis OSP, Dale PS, Plomin R. Socio-economic status (SES) and children's intelligence (IQ): In a UK-representative sample SES moderates the environmental, not genetic, effect on IQ. PLoS ONE. 2012; 7(2):e30320. Retrieved from http:// www.plosone.org/article/info\%3Adoi\%2F10.1371\%2Fjournal.pone.0030320. [PubMed: 22312423]

Haworth CMA, Wright MJ, Luciano M, Martin NG, de Geus EJC, van Beijsterveldt CEM, Plomin R. The heritability of general cognitive ability increases linearly from childhood to young adulthood. Molecular Psychiatry. 2010; 15:1112-1120. [PubMed: 19488046]

Hedges LV, Vevea JL. Fixed- and random-effects models in meta-analysis. Psychological Methods. 1998; 3:486-504.

McCartney K, Harris MJ, Bernieri F. Growing up and growing apart: A developmental meta-analysis of twin studies. Psychological Bulletin. 1990; 107:226-237. [PubMed: 2138795]

McClintock MK, Herdt G. Rethinking puberty: The development of sexual attraction. Current Directions in Psychological Science. 1996; 5:178-183.

McGue, M.; Bouchard, TJJ.; Iacono, WG.; Lykken, DT. Behavior genetics of cognitive ability: A lifespan perspective. In: Plomin, R.; McClearn, GE., editors. Nature, nurture, and psychology. Washington, DC: American Psychological Association; 1993. p. 59-76.

Muthén, LK.; Muthén, BO. Mplus user's guide. 6th. Los Angeles, CA: Author; 2010.

Neale, MC.; Cardon, LR. Methodology for genetic studies of twins and families. Dordrecht, The Netherlands: Kluwer; 1992.

Nelson CA, Zeanah CH, Fox NA, Marshall PJ, Smyke AT, Guthrie D. Cognitive recovery in socially deprived young children: The Bucharest Early Intervention Project. Science. 2007; 318:19371940. [PubMed: 18096809]

Plomin R. Multivariate analysis and developmental behavioral genetics: Developmental change as well as continuity. Behavior Genetics. 1986; 16:25-43. [PubMed: 3707486]

Plomin, R.; DeFries, JC. Origins of individual differences in infancy: The Colorado Adoption Project. Orlando, FL: Academic Press; 1985.

Plomin R, DeFries JC, Loehlin JC. Genotype-environment interaction and correlation in the analysis of human behavior. Psychological Bulletin. 1977; 84:309-322. [PubMed: 557211]

Plomin R, Spinath FM. Intelligence: Genetics, genes, and genomics. Journal of Personality and Social Psychology. 2004; 86:112-129. [PubMed: 14717631]

Scarr S, McCartney K. How people make their own environments: A theory of genotype $\rightarrow$ environment effects. Child Development. 1983; 54:424-435. [PubMed: 6683622]

Spelke ES. Sex differences in intrinsic aptitude for mathematics and science? A critical review. American Psychologist. 2005; 60:950-958. [PubMed: 16366817]

Tucker-Drob EM. Preschools reduce early academic-achievement gaps: A longitudinal twin approach. Psychological Science. 2012; 23:310-319. [PubMed: 22368155]

Tucker-Drob EM, Harden KP. Early childhood cognitive development and parental cognitive stimulation: Evidence for reciprocal gene-environment transactions. Developmental Science. 2012a; 15:250-259. [PubMed: 22356180]

Tucker-Drob EM, Harden KP. Intellectual interest mediates Gene $\times$ Socioeconomic Status interaction on adolescent academic achievement. Child Development. 2012b; 83:743-757. [PubMed: 22288554]

Turkheimer E, Waldron MC. Nonshared environment: A theoretical, methodological, and quantitative review. Psychological Bulletin. 2000; 126:78-108. [PubMed: 10668351] 


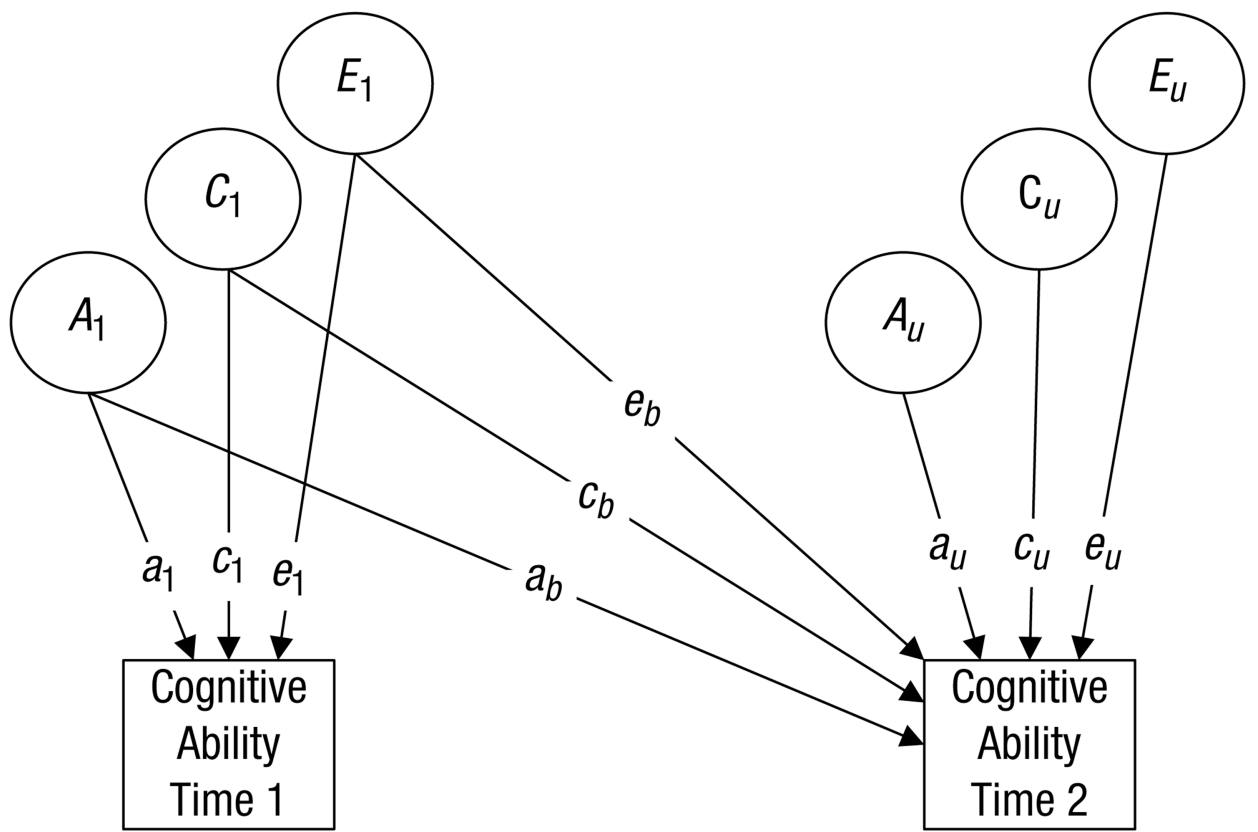

Fig. 1.

Longitudinal behavioral genetic Cholesky decomposition for one member of a sibling pair. The latent variables $A, C$, and $E$ represent the influence of genetic variation, shared environmental variation, and nonshared environmental variation, respectively. $A_{1}, C_{1}$, and $E_{1}$ represent these influences on cognition at the first measurement occasion (Time 1), and squaring the parameters $a_{1}, c_{1}$, and $e_{1}$ gives the proportion of variance in cognitive ability at Time 1 accounted for by the $A_{1}, C_{1}$, and $E_{1}$ components, respectively. The parameters labeled $a_{b}, c_{b}$, and $e_{b}$ represent the carryover of genetic and environmental effects from Time 1 to the second measurement occasion (Time 2). The latent variables $A_{u}, C_{u}$, and $E_{u}$ represent the unique variance at Time 2 , and the squared $a_{u}, c_{u}$, and $e_{u}$ parameters represent the proportion of variance attributable to novel influences. 


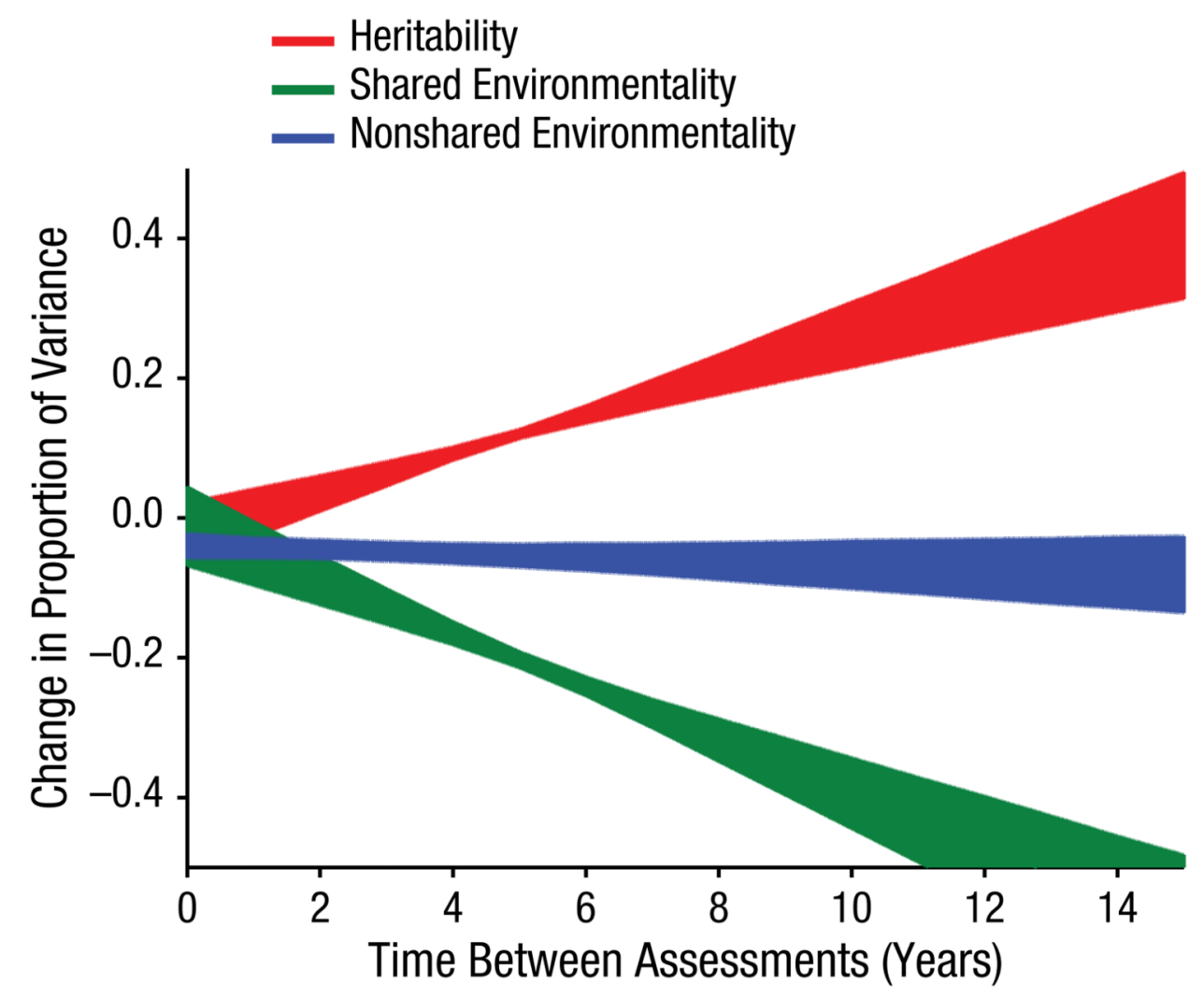

Fig. 2.

Predicted amount of change in the proportion of variance accounted for by genes, the shared environment, and the nonshared environment across time intervals between assessments. Shading represents $\pm 1 S E$. 
a

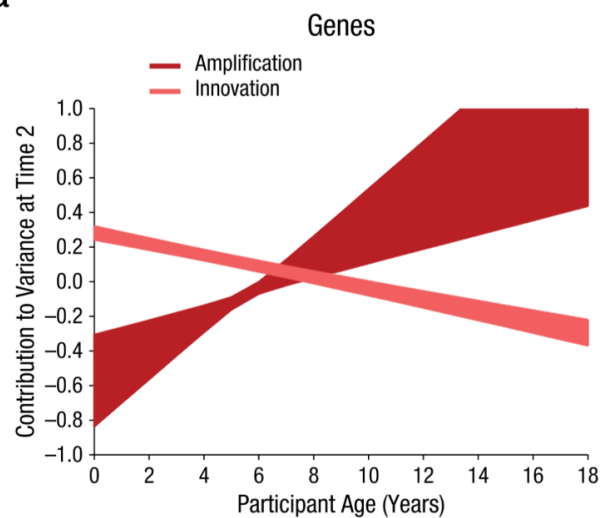

b

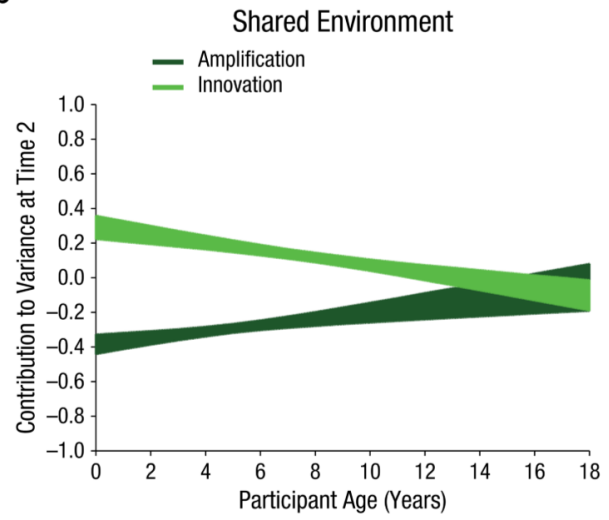

C

Nonshared Environment

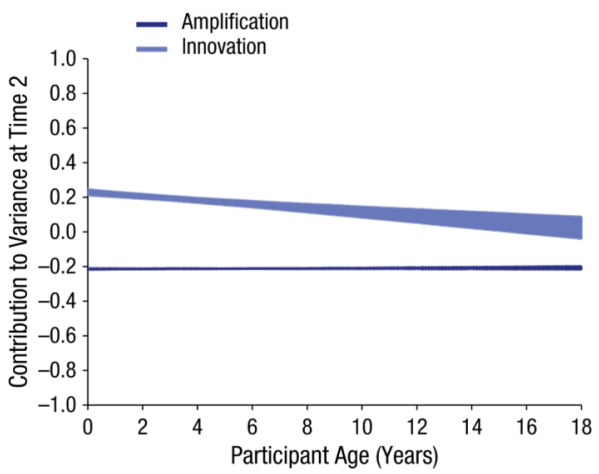

Fig. 3.

Predicted influence of amplification and innovation effects on variance in cognitive ability at the second time point of measurement across participants' age. Results are shown separately for the influence of (a) genes, (b) the shared environment, and (c) the nonshared environment. Amplification refers to the carryover of early influences across time.

Innovation refers to the emergence of novel influences over time. In each graph, shading represents $\pm 1 S E$. 
Table 1

\section{Characteristics of Participants Across Studies Included in the Meta-Analysis}

\begin{tabular}{lrrr}
\hline Variable & $\boldsymbol{M}$ & $\boldsymbol{S D}$ & Range \\
\hline Age at baseline (years) & 4.22 & 2.86 & $0.50-12.00$ \\
Time interval between measurements (years) & 4.31 & 3.38 & $0.33-15.00$ \\
Age at follow-up (years) & 8.52 & 4.33 & $1.00-18.10$ \\
Female (\%) & 50.10 & 3.27 & $47-58$ \\
Non-White (\%) & 8.45 & 1.68 & $0-39$ \\
\hline
\end{tabular}

Note: Characteristics are weighted per sample rather than by individual participants. 


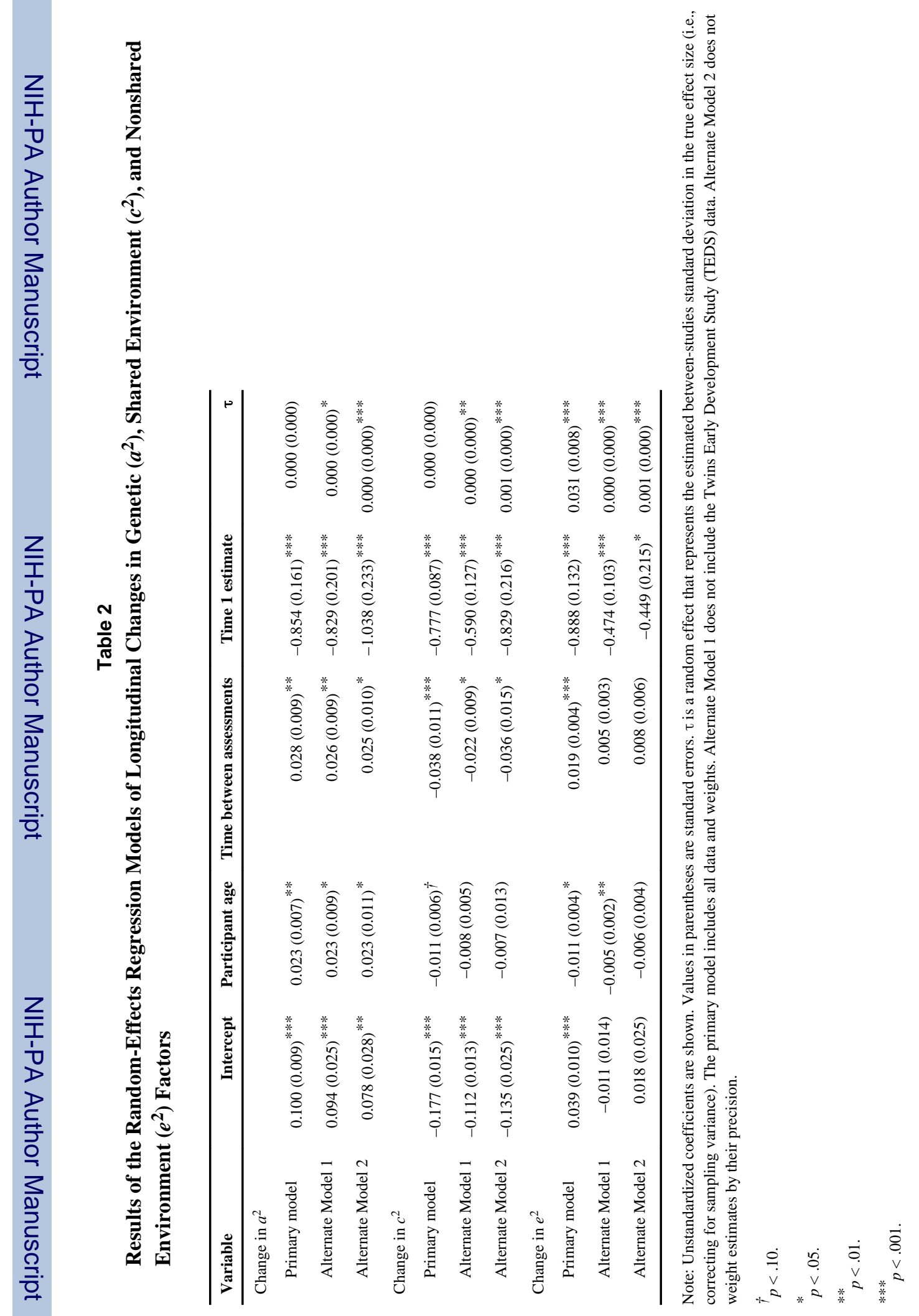

Psychol Sci. Author manuscript; available in PMC 2014 September 01. 


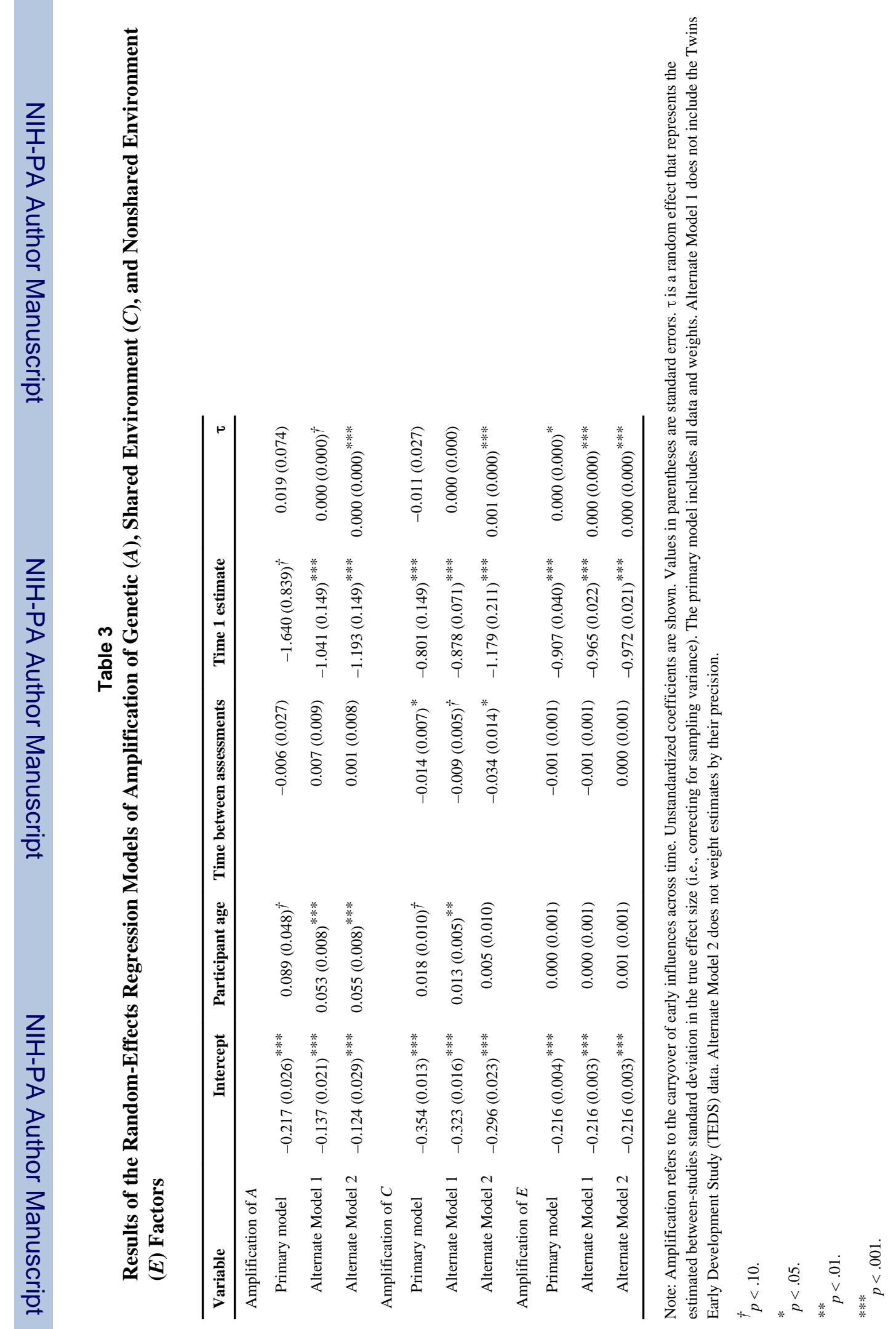

Psychol Sci. Author manuscript; available in PMC 2014 September 01. 


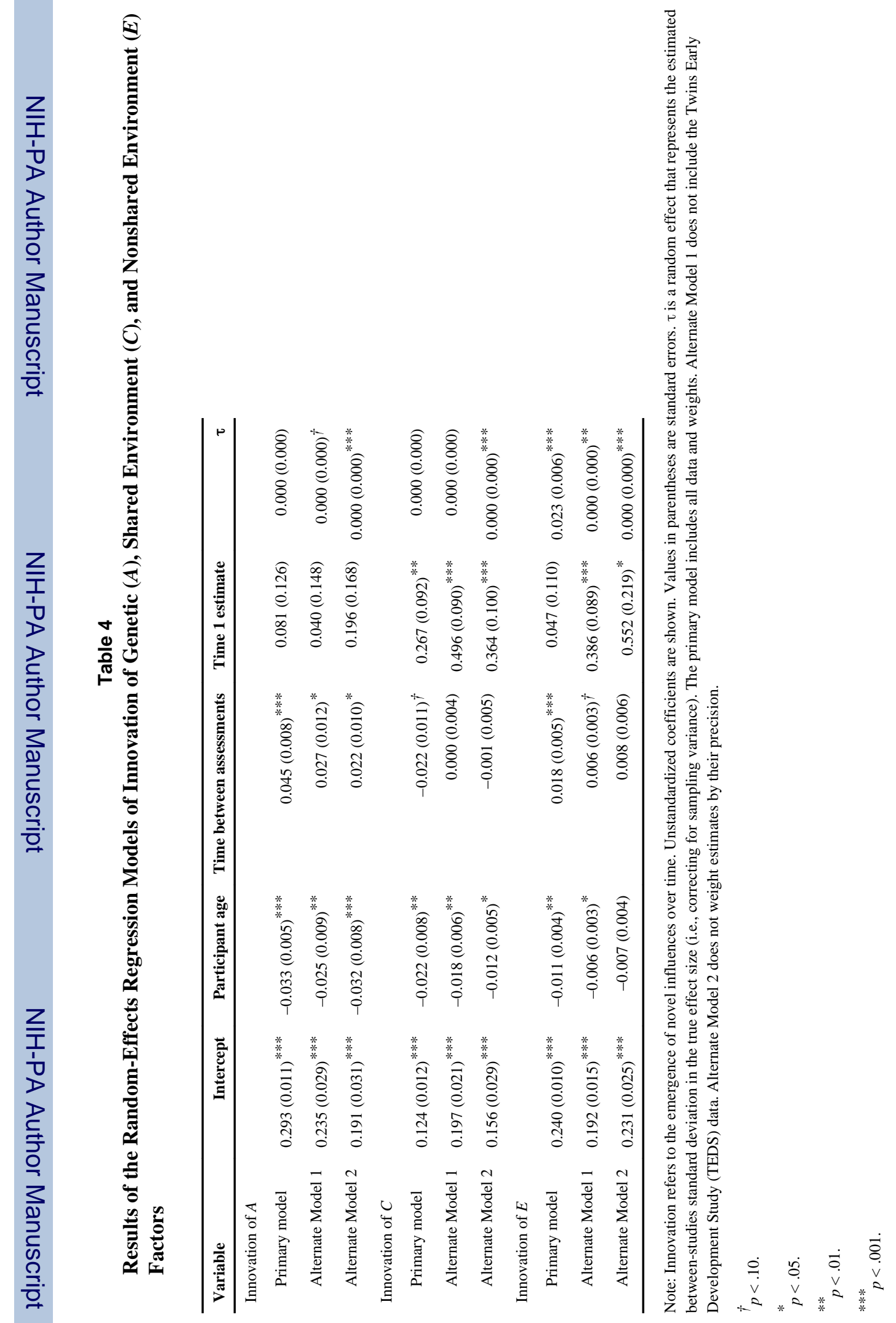

